\author{
ARTIFICIAL SATELLITES, Vol. 51, No. 1 - 2016 \\ DOI: 10.1515/arsa-2016-0004
}

\title{
A MONTE CARLO ANALYSIS FOR COLLISION RISK ASSESSMENT ON VEGA LAUNCHER PAYLOADS AND LARES SATELLITE
}

\author{
G. Sindoni \\ Sapienza Università di Roma, Scuola di Ingegneria Aerospaziale, Rome, Italy \\ e-mail: giampiero.sindoni@uniroma1.it \\ I. Ciufolini \\ Università del Salento, Dip. Ingegneria dell'Innovazione, Lecce, and Centro Fermi, Rome, Italy \\ e-mail: ignazio.ciufolini@unisalento.it \\ F. Battie \\ ELV s.p.a. \\ e-mail: francois.battie@elv.it
}

\begin{abstract}
This work has been developed in the framework of the LARES mission of the Italian Space Agency (ASI). The LARES satellite has been built to test, with high accuracy, the frame-dragging effect predicted by the theory of General Relativity, specifically the Lense-Thirring drag of its node. LARES was the main payload in the qualification flight of the European Space Agency launcher VEGA. A concern arose about the possibility of an impact between the eight secondary payloads among themselves, with LARES and with the last stage of the launcher (AVUM). An impact would have caused failure on the payloads and the production of debris in violation of the space debris mitigation measures established internationally. As an additional contribution, this study allowed the effect of the payload release on the final manoeuvers of the AVUM to be understood.
\end{abstract}

Keywords: LARES, VEGA, launchers, space debris, collisions.

\section{INTRODUCTION}

On February 13, 2012, the European Space Agency's VEGA qualification flight inserted into orbit the LARES (LAser RElativity Satellite) satellite (Ciufolini et al. (2012c); Paolozzi and Ciufolini (2013)) along with eight more small university payloads (Paolozzi et al. (2015)): one microsatellite and seven CubeSats. The high number of payloads released by VEGA gave rise to concern regarding the possibility of impact occurring with the production of more debris. Space debris is an important issue as witnessed by the continuous meetings organized by the Committee On the Peaceful Uses of Outer Space (COPUOS) of the United Nations (COPUOS (2014); Perek (2000)). VEGA is a small launcher that, along with Ariane (a large launcher) and Soyuz (a medium-sized launcher) will complete the commercialized fleet of Arianspace. VEGA has been developed by ELV (an acronym for European Launch Vehicle and an Avio and Italian Space Agency (ASI) 
joint venture) with contributions from several European countries. The VEGA launcher comprises three solid propellant stages plus one liquid propellant stage: the AVUM (Attitude and Vernier Upper Module. VEGA has the ability to bring up to two tons into low Earth orbit, from the Kourou spaceport, with any inclination between five and 105 degrees (Arianespace (2014)). The main objectives of the VEGA maiden flight were to qualify the launcher and to insert LARES into orbit. The first satellite to be released was LARES. This satellite and the mission were designed to test frame-dragging (Ciufolini et al. (2009b,c, 2010)), an intriguing phenomenon predicted by the theory of General Relativity. Frame-dragging on the node of a satellite is called the Lense-Thirring effect and was derived from General Relativity by the physicists J. Lense and H. Thirring. The idea of using two LAGEOS satellites (LAser GEodynamic Satellite) to test frame-dragging was first proposed in 1984 (Ciufolini (1984)) and later published in (Ciufolini (1986)). A number of studies had been undertaken up to the launch of LAGEOS 2 by NASA and ASI in 1992 (Ciufolini (1989); Tapley and Ciufolini (1989)). The inclination of LAGEOS 2 , however, was not ideal for the measurement of the Lense-Thirring effect. However, it was possible to reach an accuracy of approximately $10 \%$ in its measurement (Ciufolini and Pavlis (2004); Ciufolini et al. (2011); Ries (2009)). But to reach an accuracy of the order of $1 \%$ (Ciufolini et al. (2009a, 2013b)) some preliminary designs were proposed in (Ciufolini and Paolozzi (1999); Ciufolini et al. (1999, 2003)) for a new laser ranged satellite LARES. Different from its companions, LAGEOS and LAGEOS 2 that were at an altitude of about $6000 \mathrm{~km}$, the altitude of LARES was limited by the stringent constraints placed on the VEGA maiden flight, i.e., a maximum altitude of $1500 \mathrm{~km}$. This limitation, along with the need to maintain the non-gravitational perturbations as low as possible, implied a very demanding design for the satellite although inspired by the LAGEOS satellites. The main breakthrough was in choosing a tungsten alloy to manufacture the satellite body (Paolozzi et al., 2009a)). Also the single-piece design Paolozzi et al. (2011) was important to reduce the thermal thrust perturbation (Ciufolini et al. (2012b, 2014)). This design makes LARES the densest known orbiting object in the solar system and the best test particle, among artificial satellites, for tests in fundamental physics (Ciufolini et al. (2012a, 2013a)). In fact, the non-gravitational perturbations (with the exclusion of magnetic torques) are proportional to the surface-to-mass ratio which, for LARES, is the lowest ever reached by an artificial orbiting object. LARES, is completely passive and spherical, with a radius of $182 \mathrm{~mm}$ and a weight of 387 kilograms. The high density and small dimensions of the satellite forced the creation of a specific interface with the launcher (Paolozzi et al. (2009b, 2012)) in order for LARES to be as close as possible to a more conventional satellite in terms of mass distribution. In particular, the centre of mass of the satellite was required to be relatively high with respect to the launch vehicle interface. This situation called for the realization of a series of analyses to verify that in any case the release phase of the satellites could not affect the manoeuvres of the deorbiting of AVUM. The other payloads that were released were Almasat, designed by the University of Bologna, and seven CubeSats developed by other Italian universities (Sapienza and Polytechnic of Turin) as well as many more European universities, all mounted on the LARES system (Fig.1). 
Table 1: Phase of flight

\begin{tabular}{ll}
\hline Phase of flight & Orbital manoeuver \\
\hline $\begin{array}{l}\text { 2nd AVUM firing } \\
\text { Pointing manoeuver }\end{array}$ & $\begin{array}{l}\text { Reached the final orbit }\left(1450 \mathrm{~km} \times 1450 \mathrm{~km}, \mathrm{i}=69.5^{\circ}\right) \\
\text { LARES satellite separation } \\
\text { LARES satellite is released in the opposite direction to the orbital velocity, } \\
\text { the delta } \mathrm{v} \text { imposed by the separation system is } 0.75 \mathrm{~m} / \mathrm{s}\end{array}$ \\
$\begin{array}{l}\text { Despin phase } \\
\text { CCAM }\end{array}$ & $\begin{array}{l}\text { Collision and contamination avoidance manoeuver } \\
\text { Rrd AVUM firing }\end{array}$ \\
$\begin{array}{l}\text { Reached the orbital conditions to meet the space debris mitigation requirements } \\
\text { for the indirect re-entry by the decrease in the perigee } \\
\text { altitude, to reach the required disposal orbit }\left(1450 \mathrm{~km} \times 304 \mathrm{~km}, \mathrm{i}=69.5^{\circ}\right)\end{array}$ \\
$\begin{array}{l}\text { Release of secondary payloads } \\
\text { Release of microsatellites }\end{array}$ \\
Passivation phase
\end{tabular}

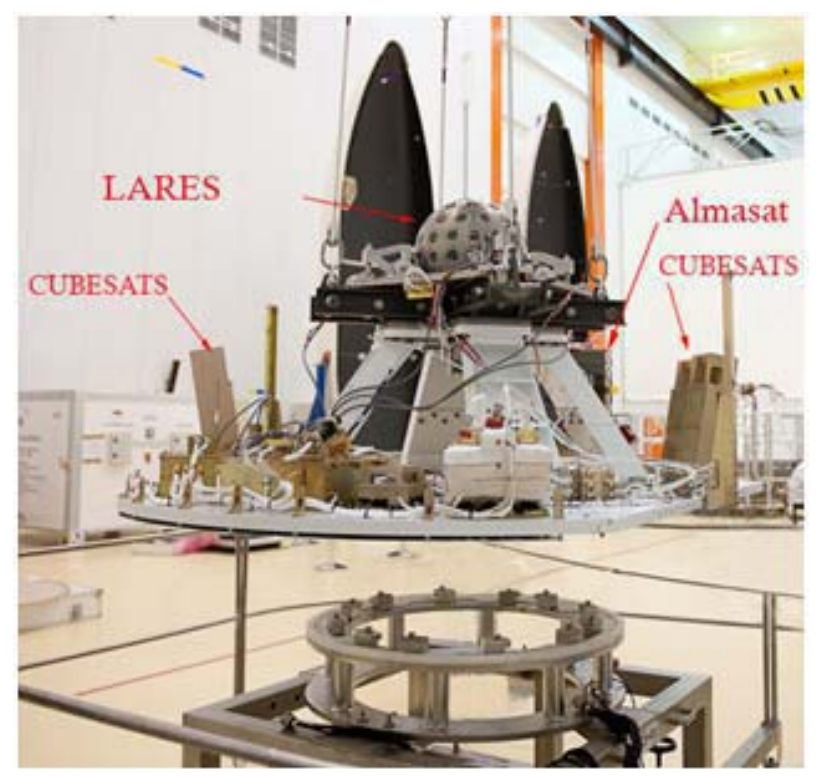

Fig. 1. LARES System.

As a consequence of the lower surface-to-mass ratio, in spite of the fact that LARES, the AVUM and the other payloads have the same apogee, impact probability with LARES rapidly drops to zero after a few orbits. In table 1 the main phases of the mission are reported.

\section{METHODOLOGY}

The probability of impacts between orbiting objects was calculated using analytical approaches well before the space era (Opik (1951/52)) using perturbation theory. The aim was to contribute to the understanding of the influence of the impacts of celestial bodies to the formation and evolution of the solar system. Unpredictably, the theory was later extended to a more practical use for studying the evolution of space debris near the orbit of the International Space Station (Rossi and Valsecchi (2006)). Another theoretical approach can be found in (Hall and Matney (2000)) based on the evolution of spatial density (Kessler (1981)). In the present work, a different point of view has been adopted due to the relatively good knowledge of the initial conditions and the relatively short orbital life of the payloads (excluding LARES). A numerical integration, undertaken using well-known and reliable orbital propagators, was therefore preferred. The number of reignitions allowed on the AVUM during the qualification flight was limited to three. This did not allow the payloads and the AVUM to be put into completely independent orbits. In fact, they all have in common the apogee. Besides being dangerous for the payloads 
themselves, a collision could create debris. This event is not acceptable under the new debris mitigation regulations (Klinkrad et al. (2004)). It was therefore mandatory, before the launch, to verify the probability of such an event. To undertake reliable analysis, the uncertainties in the initial conditions at separation of the payloads and of several other parameters affecting the payloads orbits needed to be taken into account. To produce the several sets of parameters to be used for the numerical propagation the Monte Carlo approach with both flat and Gaussian probability distributions has been chosen. Several time intervals have been taken into account: the shortest being from the release of LARES to the end of the AVUM qualification mission, i.e. one day. Medium-term analysis has been undertaken to study the evolution of the system during the first week, the long term analysis lasted for 800 days to consider the entire life time of most of the CubeSats. Before undertaking the propagations using the set of parameters provided by the Monte Carlo analysis, three nominal cases are considered, each one being different only in the perigee altitude; these cases have been taken into account because, at the time of the analysis, the mission was not completely decided. Each one of these cases is obtained by changing the nominal value of the eccentricity at the separation of the secondary payloads, i.e. at perigee. For each of these cases, the mutual distances between LARES, AVUM and the other payloads has been evaluated and finally only the case with the minimum of such distances has been retained for subsequent analysis. This has been taken as the reference case and then the main parameters affected by the uncertainties are considered: the attitude and attitude rate vectors of AVUM, the separation energy of the ejection mechanism, the ballistic characteristics $\left(C_{d}\right)$ of the payloads and AVUM and the AVUM mass variability. Two thousand sets of those parameters have been generated with flat and Gaussian distributions.

\section{INPUT DATA}

The chosen parameters have been spread using or a flat distribution or a gaussian distribution: in Fig. 2 the occurrences for the AVUM ballistic coefficient and for the pitch attitude angle are shown. The behaviour of the bar charts displays an almost gaussian distribution for the AVUM $C_{d}$ while an almost flat distribution for the AVUM mass. Similar behaviour is obtained for the other parameters that are not reported for the sake of brevity.
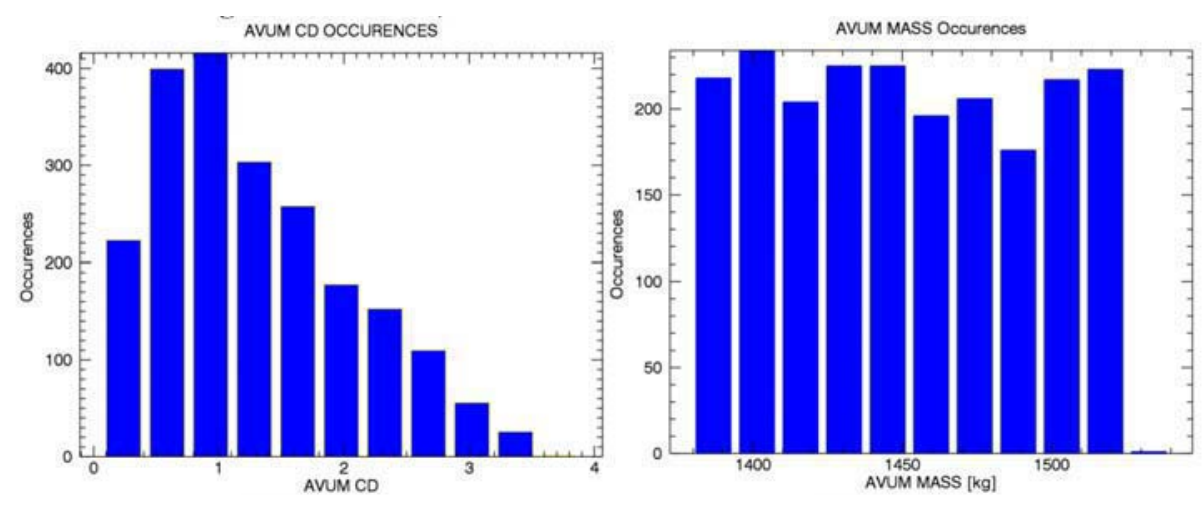

Fig. 2. Probability distributions of two AVUM parameters.

The total number of parameters used as inputs of the Monte Carlo analysis is 22, as can be verified from Table 2. The CubeSats are stowed into three different ejection 
Table 2: Spread parameters

\begin{tabular}{lllll}
\hline & AVUM & LARES & Almnasat & CubeSats ( 7) \\
\hline Attitude control & 6 & & & \\
Mass & 1 & & & 7 \\
Ballistic property & 1 & 1 & 1 & 3 \\
Ejection velocity & & 1 & 1 & \\
\hline
\end{tabular}

mechanisms (PPOD) so there are only three independent parameters for the ejection velocity. The ejection mechanism is in fact one for each PPOD. One thousand out of two thousand possible propagations have been undertaken with Geodyn, the same orbital propagator used for the data analysis of LARES. In each run, one of the 2000 sets of parameters of the previous Monte Carlo simulation was used. For each run the relative distances of any possible couples of orbiting objects have been evaluated. In the analysis, the most up to date models available in Geodyn have been used: the gravity field model with order and degree up to 90, with time dependent parts up to 30x30 with tidal effect 60x60. The atmospheric effects have been taken into account using the French Drag Model for the nominal cases, while in the other analysis both Jacchia 1971 and MSIS Empirical Drag models have been used, the ephemerides of all the planets have been considered to improve the orbital propagation accuracy, and also direct solar and reflected radiation pressure have been implemented. The integration steps have been chosen as follows: 0.1 sec for the short term analysis, $10 \mathrm{sec}$ for the medium term analysis and $50 \mathrm{sec}$ for the 800 days analysis.

\section{SUCCESS CRITERIA}

Two objects were considered to be in collision when the relative distance was less than 10 metres in the short term analysis while during the long term analysis the threshold was set to 500 metres. For collision risk assessment the energy-to-mass ratio (EMR) has been introduced as:

$$
E M R=\frac{0.5 M_{p} v_{i m p}^{2}}{M_{t}}
$$

where $v_{i m p}$ is the relative velocity, $M_{t}$ is the target mass and $M_{p}$ is the impactor mass. When $E M R$ is less than $40000 \mathrm{~J} / \mathrm{kg}$ it is assumed that debris is not created. The amount of data produced during the simulations was of the order of two gigabytes per run and per spacecraft, so that the total amount would have been more than 20 terabytes and, therefore, it was considered inconvenient to store all the data. The runs were undertaken in a cluster of four machines using 36 processors. The time required for the propagations was three weeks, 24 hours per day. The data have been used to calculate the minimum distances between each pair of payloads; in fact, of concern are the occurrences of the minimum distances rather than the full evolution of the distances in time. Once this had been calculated the full data was deleted. For illustrative purposes, the behaviour in the nominal case of the distances of five objects as a function of time for the first few hours after release is shown in Fig. 3. LARES is not reported because it is in a much different orbit with only the apogee in common and therefore the distances to any other spacecraft would, for most of the time, have been thousands of kilometres. 
Table 3: Osculating Keplerian orbital parameters

\begin{tabular}{llllllll}
\hline SAT & Date & A $(\mathrm{m})$ & $\mathrm{E}$ & $\mathrm{I}(\mathrm{deg})$ & Asc Node(deg) & Perigee (deg) & Mean anomaly (deg) \\
\hline AVUM & 12072443716.1 & 7247144.570 & 0.0776 & 69.5246 & 247.60 & 169.67 & 91.7 \\
Almasat & 12072443716.1 & 7237907.276 & 0.0770 & 69.523 & 246.49 & 169.09 & 92.8 \\
\hline
\end{tabular}

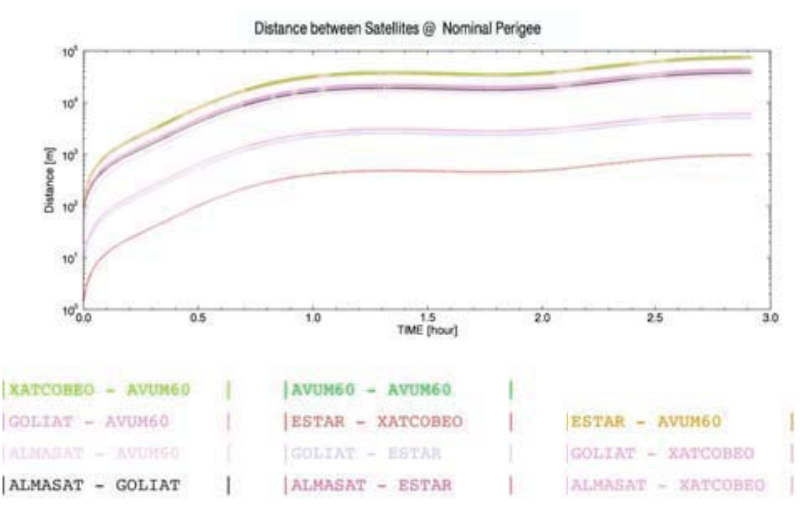

Fig. 3. Time history of mutual distances between three CubeSats, Almasat and AVUM.

The data plotted are extracted from the short term analysis which is for 24 hours. The minimum distance in this time span was only a few metres between two CubeSats but just immediately after separation; the EMR has been evaluated in all cases but the threshold of $40000 \mathrm{~J} / \mathrm{Kg}$ was not execeeded: the behavour of the EMR is shown in Fig. 4 .

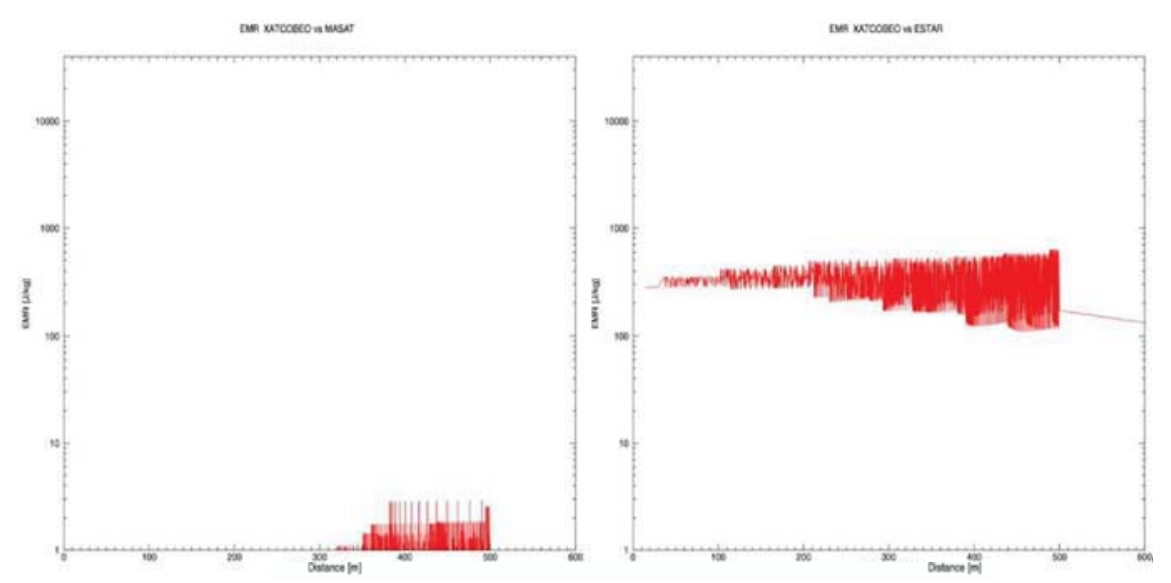

Fig. 4. Energy-to-mass ratio (EMR) for two different couples of CubeSats.

But if one looks at the following 800 days (long-term analysis) the minimum distance was found to be between AVUM and Almasat, with a value of about $1.3 \mathrm{~km}$ after six months; this result, indeed unexpected, (before the analysis, the minimum distance was expected to be found between two CubeSats) is caused by just a coincidence in the relative positions of the spacecraft. In Table 1 the osculating Keplerian orbit at the time of the closest approach is shown.

The difference between the two semi-major axes is about $10 \mathrm{~km}$, which is consistent with the decreasing ratio of the semi-major axis of Almasat at around $22 \mathrm{~km} /$ year, and for the AVUM at around $4 \mathrm{~km} /$ year. However, the combination of the slight rotation of the argument of the perigee and the slight difference in the mean motion brings Almasat in a position closer to the AVUM than expected. 


\section{RESULTS}

The results of the simulations driven by the Monte Carlo analysis have been summarized in Fig. 5. For the sake of brevity, just the results of the long term analysis are reported. In the graph, for all runs, the number of times the relative distances between two satellites are in the specified intervals is reported. The number of rows in the following table is equal to the number of possible combinations of two payloads drawn from the eight payloads plus LARES and the AVUM. In other words, the number of rows is equal to the binomial coefficient:

$$
\left(\begin{array}{c}
10 \\
2
\end{array}\right)=\frac{10 !}{(10-2) ! 2 !}=45
$$

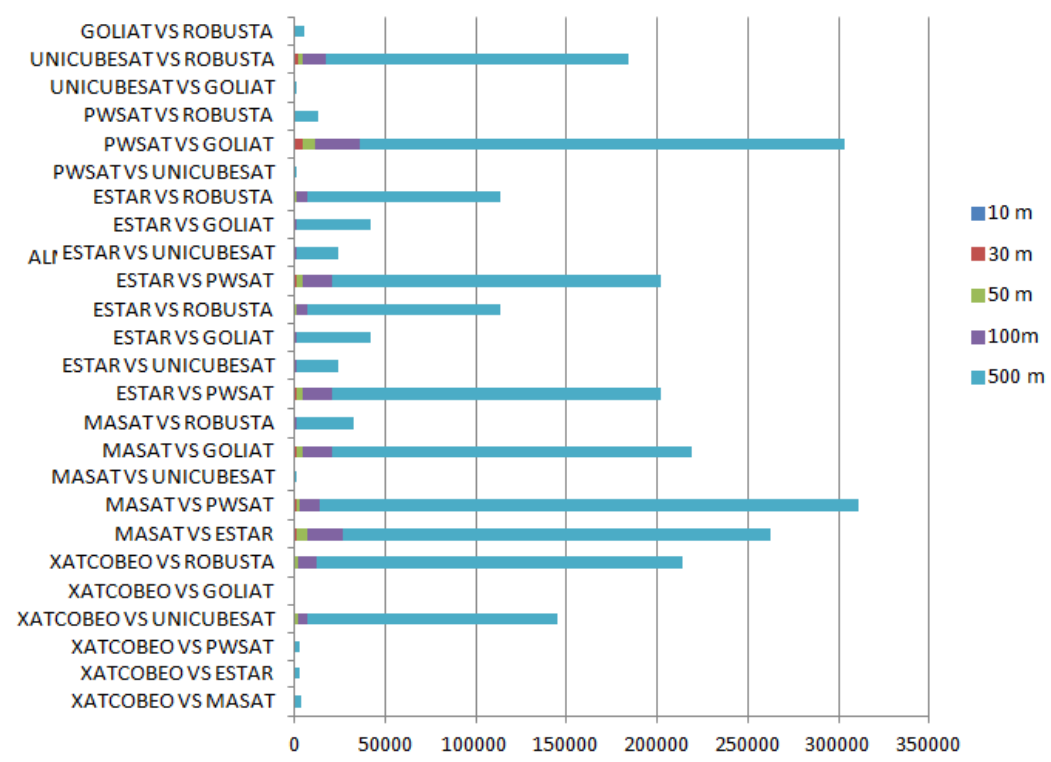

Fig. 5. Bar plot.

In the presented results of the Monte Carlo analysis, it is possible to see that during the "long-term" analysis the orbiting objects are at risk of collision a huge number of times; however, in the event of a collision, there is no associated danger because of the low values of the EMR: in the case in which the distance was below the threshold, the impact energy was negligible. For the sake of brevity the behaviours of the EMR are not reported in this case. This result can be intuitively explained using the following two arguments:

- larger orbiting objects (LARES, AVUM, Almasat) have orbits that lie far away from one another as the objects are very different in mass, size and surface properties

- CubeSats orbits are closer since the geometries of the CubeSats are quite similar to one another and consequently their orbits are confined in a narrower band: this causes thousands of instances in which distances among the cubesats are lower than the assigned threshold, but, on the other hand, the orbits themselves are so close that it is practically impossible for a colliding pair of cubesats to have enough energy to create debris. 


\section{CONCLUSIONS}

In the paper, a collision risk assessment has been undertaken, using orbital propagators, among all the payloads including the last stage of the VEGA launcher. The results have shown that no collision risk was present during the VEGA maiden flight. The successful launch of VEGA on $13^{\text {th }}$ February 2012 has confirmed the short-term analysis undertaken. The ongoing measurement campaign, that is still running with LARES, also shows the consistency of the long-term analysis.

Acknowledgments. The authors wish to thank the Italian Space Agency for supporting the LARES mission, ELV for sponsoring the present study, ESA for providing the VEGA qualification launch and the International Laser Raging Service for providing LARES tracking.

\section{REFERENCES}

Arianespace. VEGA user's manual, April 2014. Issue 4 - Revision 0.

I. Ciufolini. Theory and experiments in general relativity and in others metric theories. PhD thesis, University of Texas at Austin, USA, 1984.

I. Ciufolini. Measurement of the Lense-Thirring drag on high-altitude laser-ranged artificial satellites. Physical Review Letters, 56:278-281, 1986.

I. Ciufolini. A comprehensive introduction to the LAGEOS gravitomagnetic experiment: from the importance of the gravitomagnetic field in physics to preliminary error analysis and error budget. Int. J. Mod. Phys. A, 4:3083-3145, 1989.

I. Ciufolini and A. Paolozzi. LARES: a new laser ranged satellite for fundamental physics and general relativity. Actual Problems of Aviation and Aerospace Systems, 1:61-73, 1999 .

I. Ciufolini and E. C. Pavlis. A confirmation of the general relativistic prediction of the Lense-Thirring effect. Nature, 431:958-960, 2004.

I. Ciufolini, A. Paolozzi, I. Peroni, and A. Gabrielli. A study for a laser ranged relativity satellite. In Proceedings of Sixth Pan American Congress of Applied Mechanics and Eighth International Conference on Dynamics Problems in Mechanics, PACAM VI/DINAME 99, Rio de Janeiro, Brasil, pages 467-470, 4-8 January 1999.

I. Ciufolini, D.G. Currie, and A. Paolozzi. The LARES mission for testing the dynamics of general relativity. In IEEE Aerospace Conference, Big Sky, Montana, USA. IEEE, 5-12 March 2003.

I. Ciufolini, A. Paolozzi, E.C. Pavlis, J.C. Ries, R. Koenig, R.A. Matzner, G. Sindoni, and H. Neumayer. Towards a one percent measurement of frame dragging by spin with satellite laser ranging to LAGEOS, LAGEOS 2 and LARES and GRACE gravity models. Space Science Reviews, 148(1-4):71-104, 2009a.

I. Ciufolini, A. Paolozzi, G. Sindoni, E.C. Pavlis, and A. Gabrielli. Scientific aspects of LARES mission. In 60th International Astronautical Congress - IAC, Daejeon, Republic of Korea, number IAC-09.B4.2.9. IAF, 12-16 October 2009b. 
I. Ciufolini, A. Paolozzi, G. Sindoni, E.C. Pavlis, A. Gabrielli, S. Pirrotta, D. Barbagallo, and E. Mangraviti. Objective of LARES satellite. In XX congresso nazionale AIDAA, Milano, Italy. Associazione Italiana di Aeronautica e Astronautica, 29 June - 3 July 2009c.

I. Ciufolini, A. Paolozzi, E. C. Pavlis, G. Sindoni, R. Koenig, J. Ries, and R. Matzner. The LARES space experiment: LARES orbit, error analysis and satellite structure. In I. Ciufolini and R. A. Matzner, editors, General Relativity and John Archibald Wheeler, volume 367 of Astrophysics and Space Science Library. Springer, 2010.

I. Ciufolini, A. Paolozzi, E. C. Pavlis, J. Ries, R. Koenig, R. Matzner, G. Sindoni, and H. Neumayer. Testing gravitational physics with satellite laser ranging. The European Physical Journal Plus, 126(8):72, 2011. doi: 10.1140/epjp/i2011-11072-2.

I. Ciufolini, A. Paolozzi, and C. Paris. Overview of the LARES mission: orbit, error analysis and technological aspects. Journal of Physics. Conference Series, 354, 2012a. conference 1 .

I. Ciufolini, A. Paolozzi, E. Pavlis, J. Ries, V. Gurzadyan, R. Koenig, R. Matzner, R. Penrose, and G. Sindoni. Testing general relativity and gravitational physics using LARES satellite. The European Physical Journal Plus, 127:133, 2012b.

I. Ciufolini, A. Paolozzi, E. Pavlis, J. Ries, R. Koenig, R. Matzner, V. Slabinski, V. Gurzadyan, E. Flamini, G. Sindoni, C. Paris, and H. Neumayer. Initial orbit determination results for the LARES satellite. In 63rd International Astronautical Congress IAC 2012, Naples, Italy. IAF, October 2012c.

I. Ciufolini, V. Gurzadyan, R. Penrose, and A. Paolozzi. Geodesic motion in general relativity: LARES in earth's gravity. In V. Gurzadyan, A. G. Sedrakyan, and A. Klumper, editors, Low Dimensional Physics and Gauge Principles, pages 1-4. World Scientific, 2013a.

I. Ciufolini, B. Moreno Monge, A. Paolozzi, R. Koenig, G. Sindoni, G. Michalak, and E. Pavlis. Monte carlo simulations of the LARES space experiment to test general relativity and fundamental physics. Classical and Quantum Gravity, 30, 2013b.

I. Ciufolini, A. Paolozzi, C. Paris, and G. Sindoni. The LARES satellite and its minimization of the thermal forces. In IEEE International Workshop on Metrology for Aerospace - MetroAerospace, Benevento, Italy, May 29-30 2014.

COPUOS. Report of the scientic and technical subcommittee on its fty-rst session, vienna, from 10 to 21 February 2014.

D.T. Hall and M.J. Matney. Collision probabilities for keplerian orbits. Space Debris, 2: 161-198, 2000.

D.J. Kessler. Derivation of the collision probability between orbiting objects: the lifetime of jupiters outer moons. Icarus, 48:39-48, 1981.

H. Klinkrad, P. Beltrami, S. Hauptmann, C. Martin, H. Sdunnus, H. Stokes, R. Walker, and J. Wilkinson. The \{ESA\} space debris mitigation handbook 2002. Advances in Space Research, 34(5):1251 - 1259, 2004. 
E.J. Opik. Collision probabilities with the planets and the distribution of interplanetary matter. In Proceedings of the Royal Irish Academy. Section A: Mathematical and Physical Sciences, volume 54, 1951/52.

A. Paolozzi and I. Ciufolini. LARES succesfully launched in orbit: satellite and mission description. Acta Astronautica, 91:313-321, 2013.

A. Paolozzi, I. Ciufolini, C. Vendittozzi, and F. Felli.

A. Paolozzi, I. Ciufolini, F. Felli, A. Brotzu, and D. Pilone. Issues on LARES satellite material. In 60th International Astronautical Congress - IAC, Daejeon, Republic of Korea, number IAC-09.C2.4.5. IAF, 12-16 October 2009a.

A. Paolozzi, I. Ciufolini, C. Paris, G. Sindoni, M. Ramiconi, F.M. Onorati, and L. Scolamiero. Design of LARES separation system. In XX congresso nazionale AIDAA, Milano, Italy. Associazione Italiana di Aeronautica e Astronautica, 29 June - 3 July 2009b.

A. Paolozzi, I. Ciufolini, and C. Vendittozzi. Engineering and scientific aspects of LARES satellite. Acta Astronautica, 69:127-134, 2011.

A. Paolozzi, I. Ciufolini, F. Passeggio, G. Caputo, L. Caputo, A. Bursi, and E. Mangraviti. LARES satellite and separation system. In 63rd International Austronautical Congress IAC 2012, Naples, Italy. IAF, October 2012.

A. Paolozzi, I. Ciufolini, C. Paris, and G. Sindoni. LARES, a new satellite specifically designed for testing general relativity. International Journal of Aerospace Engineering, Volume 2015:9 pages, 2015. Article ID 341384.

L. Perek. Space debris at the united nations. Space Debris, 2:123-136, 2000.

J. C. Ries. Relativity in satellite laser ranging. In IAU Symposium 261. Relativity in Fundamental Astronomy: Dynamics, Reference Frames, and Data Analysis. Virginia Beach, VA, USA. IAU, 27 April - 1 May 2009.

A. Rossi and G.B. Valsecchi. Collision risk against space debris in earth orbits. Celestial Mechanics and Dynamical Astronomy, 95:345-356, 2006.

B.D. Tapley and I. Ciufolini. Measuring the Lense-Thirring precession using a second LAGEOS satellite. Technical report, Center for Space Research - University of Texas at Austin, 1989. CSR-UT publication n. CSR-89-3.

Received: 2015-11-05,

Reviewed: 2015-12-30, by E. Pavlis,

Accepted: 2016-02-01. 